\title{
Cognitive Performance of MDMA-Treated Rhesus Monkeys: Sensitivity to Serotonergic Challenge
}

Michael A. Taffe, Ph.D., Sophia A. Davis, B.S., Jie Yuan, M.D., Richard Schroeder, M.S., George Hatzidimitriou, Ph.D., Loren H. Parsons, Ph.D., George A. Ricaurte, M.D., Ph.D., and Lisa H. Gold, Ph.D.

Recreational users of $( \pm) 3,4-$ methylenedioxymethamphetamine (MDMA, "Ecstasy") exhibit poor performance on a number of neurocognitive measures, with tests of memory and attention most commonly affected. Cognitive impairments can be persistent or possibly permanent, since users who have been abstinent from MDMA for many months are also impaired. Repeated treatment of rats or nonhuman primates with MDMA has consistently been demonstrated to produce specific, lasting depletions of brain serotonin (5-HT) markers, a potential source of such cognitive symptoms. We have shown, however, that monkeys treated with a regimen of MDMA (4 days, $10 \mathrm{mg} / \mathrm{kg}$ i.m., b.i.d.), sufficient to produce a $50 \%$ reduction of the 5-HT metabolite 5-hydroxyindoleacetic acid in cerebrospinal fluid, do not exhibit lasting deficits in a range of cognitive domains. Acute drug challenges are often effective at unmasking consequences of amphetamine toxicity. Here, the performance of MDMA-treated and control monkeys on tests of spatial working memory (self- ordered spatial search), vigilance and reaction time (5-choice reaction time), reinforcer efficacy and sustained attention (progressive ratio responding) and fine motor control (bimanual motor skill task) was challenged with ketanserin (0.1-1.7 mg/kg, i.m.), 1-(3-Chlorophenyl)piperazine dihydrochloride $(\mathrm{mCPP}, 0.03-0.5 \mathrm{mg} / \mathrm{kg}$, i.m.) and $( \pm) 8$ hydroxy-DPAT hydrobromide (8-OH-DPAT, 0.032-0.1 mg/ $\mathrm{kg}$, i.m.). MDMA-exposed animals exhibited increased sensitivity to challenge with $m C P P$ on the reaction time and progressive ratio tasks but otherwise were equivalently sensitive to drug challenge. Post-mortem analysis demonstrated that 76-93\% reductions of 5-HT in neocortex persist 17-20 months post-MDMA. These observations suggest that large depletions of brain 5-HT produced by MDMA can persistently alter behavioral sensitivity to the disrupting effects of serotonergic agents.

[Neuropsychopharmacology 27:993-1005, 2002] (C) 2002 American College of Neuropsychopharmacology. Published by Elsevier Science Inc.
From the Department of Neuropharmacology, The Scripps Research Institute, La Jolla, CA (MAT, SAD, RS, LHP, LHG), and the Department of Neurology, Johns Hopkins Medical Institutions, Baltimore, MD (JY, GH, GAR).

Address correspondence to: Dr. Michael A. Taffe, Department of Neuropharmacology, CVN-7, The Scripps Research Institute, La Jolla, CA 92037. Tel.: (858) 784-7228; E-mail: mtaffe@scripps.edu

Present Address for L.H. Gold: Pharmacia Corporation, Mail code 7251-209-405, 301 Henrietta Street, Kalamazoo, MI 49007.

Received January 16, 2002; revised May 22, 2002; accepted May 29, 2002.

Online publication: 5/30/02 at www.acnp.org/citations/ Npp053002315/default.htm.
KEY WORDS: MDMA; Attention; Memory; Ketanserin; $m C P P ; 8-O H-D P A T$

Recreational use of ( \pm )3,4-methylenedioxymethamphetamine (MDMA, "Ecstasy") has become increasingly popular in recent decades (Peroutka 1987; Pope et al. 2001; Schuster et al. 1998) and use continues to accelerate. Surveys of drug use in US adolescents and college students report steep increases in the rate of MDMA use while use of a number of other recreational drugs has been stable or in decline (Johnston et al. 
2001a,b; Pope et al. 2001). Surveys from other countries also indicate substantial rates of MDMA use (Abraham et al. 1998; Hibell et al. 2000; Ramsey and Spiller 1997). As the popularity of MDMA grew in the 1980s and 1990s, preclinical research demonstrated that MDMA exposure can result in persistent and possibly permanent alterations in serotonin (5-HT) neurons of the central nervous system of New World and Old World monkeys as well as rodents (see McKenna and Peroutka 1990; Ricaurte et al. 2000; Steele et al. 1994 for review). Recent studies have now shown with minimally invasive imaging techniques that similar 5-HT alterations are present in abstinent human users of MDMA (McCann et al. 1998; Reneman et al. 2000; Reneman et al. 2001; Semple et al. 1999). These observations alone present the strong possibility that recreational use of MDMA may result in lasting adverse consequences for brain function.

Concern regarding possible functional consequences of MDMA exposure has been supported by recent studies demonstrating that frequent recreational users of MDMA exhibit poor performance on neurocognitive measures, e.g., (Bhattachary and Powell 2001; Gouzoulis-Mayfrank et al. 2000; McCann et al. 1999; Morgan 1999; Rodgers 2000; Verkes et al. 2001; Zakzanis and Young 2001), with tests of memory and attention most commonly affected (also see Morgan (2000) and Parrott (2000) for review). Such cognitive impairments may be persistent or possibly permanent, since even users who have been abstinent from MDMA for many months have been shown to be impaired relative to poly-drug using controls (Bhattachary and Powell 2001; Gouzoulis-Mayfrank et al. 2000; McCann et al. 1999; Morgan 1999). Since repeated treatment of nonhuman primates with MDMA has consistently been demonstrated to produce lasting and selective depletions of brain 5-HT markers (Fischer et al. 1995; Insel et al. 1989; Ricaurte et al. 1988b, 1992), the most obvious working hypothesis is that the demonstrated alterations of normal 5-HT transmission in abstinent human users may cause cognitive symptoms. This hypothesis is supported more specifically by evidence that cognitive deficits in abstinent MDMA users are correlated with the concentration of the 5-HT metabolite 5-hydroxyindoleacetic acid (5-HIAA) in cerebrospinal fluid (CSF) samples (McCann et al. 1999) as well as evidence that memory performance in abstinent MDMA users is positively correlated with $5-\mathrm{HT}_{2 \mathrm{~A}}$ binding in cortex (Reneman et al. 2000).

Although the available evidence from studies of human MDMA users is suggestive, such studies cannot easily demonstrate a direct relationship between MDMA-related alterations of serotonergic neurotransmission and cognitive impairment. The MDMA user populations studied are typically multi-drug users with considerable prior and continuing exposure to cannabis and alcohol, among other substances, which may themselves produce lasting negative effects on cognition. Indeed recent studies provide evidence that memory deficits observed in MDMA users are either likely (Croft et al. 2001) or unlikely (Rodgers 2000) to be attributable to concurrent use of cannabis. Certainly, study of "pure" MDMA-abusing populations is difficult if not impossible and therefore efforts to directly link MDMA-associated alterations of central nervouse system (CNS) 5-HT systems require experimental studies conducted in nonhuman models. Such model systems also provide advantages in specifically controlling the MDMA dose, exposure regimen, and timing of behavioral evaluation relative to prior exposure which may help to interpret or explain findings from human studies.

We have previously shown that rhesus monkeys treated with a high-dose repeated regimen of MDMA (4 days, $10 \mathrm{mg} / \mathrm{kg}$ i.m., b.i.d.) sufficient to produce $\sim 50 \%$ reduction of the 5-HT metabolite 5-hydroxyindoleacetic acid (5-HIAA) in cerebrospinal fluid do not exhibit performance deficits in a range of cognitive domains (Taffe et al. 2001). Those results were consistent with a similar investigations employing different behavioral assays in both rhesus (Frederick et al. 1998) and squirrel monkeys (Winsauer et al. 2002). In our previous work we showed that the CSF 5-HIAA concentrations in the MDMA-exposed animals recovered to pre-treatment values by approximately five months post-MDMA. Consistent with this observation, other studies have shown that nonhuman primates exposed to repeated MDMA may exhibit a degree of serotonergic recovery and/or reinnervation over a time course of weeks to months post-exposure (Fischer et al. 1995; Hatzidimitriou et al. 1999). Such recovery of 5-HT systems may be selective since restoration of 5-HT function/structure in subcortical structures was more common and extensive in comparison to that reported in cortical regions. Consequently it is likely that cognitive and performance functions that depend on cortical structures may be more sensitive to further 5-HT perturbations in MDMA-exposed monkeys, in comparison to those that depend on subcortical structures.

The purpose of the present study was therefore to determine if the monkeys treated with a high-dose repeated MDMA regimen in our previous study exhibited increased sensitivity to the cognitive effects of challenge with serotonergic agents. Consequently, the MDMA-treated monkeys' and vehicle-treated control monkeys' performance on tests of memory, reaction time, reinforcer efficacy and fine motor control was challenged serially with doses of the mixed $5 \mathrm{HT}_{2 \mathrm{~A} / 2 \mathrm{C}}$ antagonist ketanserin, the $5 \mathrm{HT}_{2 \mathrm{C}}$ agonist 1-(3-Chlorophenyl)piperazine dihydrochloride ( $\mathrm{mCPP}$ ) and the $5 \mathrm{HT}_{1 \mathrm{~A}}$ agonist $( \pm) 8$-hydroxy-DPAT hydrobromide (8$\mathrm{OH}$-DPAT). These compounds were chosen to probe serotonergic sensitivity because each has been demon- 
strated to disrupt behavioral responding in nonhuman primates (DeNoble et al. 1991; McKearney 1990; Winsauer et al. 1996). It was hypothesized that the performance of MDMA-exposed monkeys would be more sensitive to the disrupting effects of these compounds because of an underlying compromise of 5-HT system integrity.

\section{METHODS}

\section{Subjects}

Six adult male rhesus monkeys (Macacca mulatta) served as subjects. The monkeys were approximately six years of age and weighed $6-8 \mathrm{~kg}$ at the beginning of the study. Animals were individually housed and fed in the home cage after completion of the daily testing session. The animals' normal diet (Lab Diet 5045, PMI Nutrition International) was supplemented with fruit or vegetables four days per week and water was available ad libitum in the home cage at all times. Principles of laboratory animal care (Clark et al. 1996) were followed, and all protocols were approved by the Institutional Animal Care and Use Committee of The Scripps Research Institute. The monkeys were obtained in a single cohort from the same vendor and received identical training on all components of the behavioral test battery prior to the initiation of the MDMA exposure study.

\section{MDMA Exposure}

Three of the monkeys were exposed to a repeated, highdose regimen of $( \pm) 3$,4-methylenedioxymethamphetamine $\mathrm{HCl}$ (MDMA) (4 days, $10 \mathrm{mg} / \mathrm{kg}$ i.m., b.i.d., expressed as the salt) 13 months prior to beginning of the present study as has been previously described (Taffe et al. 2001). The remaining three animals were treated with vehicle injections on the same schedule and serve as control subjects. The MDMA regimen resulted in an initial reduction $(\sim 50 \%)$ in CSF concentrations of the 5-HT metabolite 5-hydroxyindoleacetic acid (5-HIAA) in CSF samples obtained 2-17 weeks post-MDMA when compared with pre-treatment values and control subjects. These results are consistent with previous studies in which analysis of CSF 5-HIAA concentrations was combined with more exhaustive post-mortem analysis of monoamine tissue concentrations (Insel et al. 1989; Ricaurte et al. 1988a, 1992).

\section{Behavioral Testing}

Cognitive and behavioral performance was evaluated using a battery of tests designed for neuropsychological testing of rhesus monkeys which has been previously described (Weed et al. 1999). The battery included tests of memory (self-ordered spatial search; SOSS), sustained attention and reinforcer efficacy (progressive ratio; PR), reaction time (RT) and bimanual motor coordination (Bimanual). Performance on tests of this battery has been shown to be sensitive to acute challenge with psychoactive compounds (Taffe et al. 1999; Weed and Gold 1998).

Apparatus. Animals were transferred to the testing room in transport cages $(80 \mathrm{~cm}$ high $\times 60 \mathrm{~cm}$ wide $\times 70$ $\mathrm{cm}$ deep) similar to the home cage but modified by the removal of several bar sections from the front to allow the animal to easily reach out of the cage. The transport cage was placed in front of a computer monitor fitted with a touch-sensitive screen on which visual stimuli were presented. The animal was trained to reach out of the cage to touch the location on the screen at which visual stimuli were presented to obtain a food pellet reinforcer. Stimulus presentation and response detection were controlled by a micro-computer equipped with a version of CANTAB (CAmbridge Neuropsychological Test Automated Battery, CeNeS Pharmaceuticals, pfc, Cambridge, UK) designed for use with non-human primates. A dispenser delivered 190-mg flavored pellets (P.J. Noyes Co., Lancaster, NH) to a bin mounted on the front of the cage after correct responses. A white-noise generator positioned just behind the touchscreen was turned on during each behavioral session.

Test Battery. The test battery employed consists of four behavioral tasks. Three of the tasks are part of the non-human primate CANTAB and require monkeys to respond by touching the touch-sensitive computer screen to obtain food pellet reinforcers. The fourth task requires the animal to extract raisins from holes in a transparent plastic board.

Self-ordered Spatial Search. In each trial of the SOSS task, two, four, six, or eight small colored rectangles (boxes) were displayed on the screen in positions randomly allocated from among 16 possible locations. The animal was required to touch a box within $30 \mathrm{~s}$ of stimulus onset. After each successful touch, the color of the touched box was briefly (100 ms) changed and then the screen was blanked and a reinforcer is delivered. After a 2-s delay, the boxes were re-displayed and the animal was required to touch a box which had not previously been touched in the trial to obtain the next reinforcer. The trial was completed when the animal had either touched all boxes without a repetition (correct), touched a box that had previously been selected in that trial (error) or failed to touch a box within $30 \mathrm{~s}$ of stimulus presentation (omission). Errors and omissions were followed by a tone and a 4-s screen blank. After an inter-trial interval of $5 \mathrm{~s}$, another trial was presented with stimuli in new (randomly allocated) positions. A session consisted of 40 trials grouped into eight blocks by 
trial type as follows: 5 ( 2 boxes), 5 ( 4 boxes), 5 ( 6 boxes), 5 (8 boxes), 5 (4 boxes), 5 (6 boxes), 5 ( 8 boxes), 5 (2 boxes). Accuracy scores were calculated for each trial type by dividing the number of correctly completed trials by the number of trials in which there was at least one response (i.e., errors of omission were excluded from the calculation).

Five-choice Reaction Time. For the RT task a response lever (BRS/LVE, Laurel, MD) was mounted below the monitor and in front of the transport cage. For each trial, a grid of five circles, or target locations, connected by lines was presented in white on the screen. The monkey initiated the trial by holding down the lever. After a pseudorandomly-variable delay lasting between 0.75 and $2.5 \mathrm{~s}$, a yellow circle appeared within one of the five target locations and was removed after 20,100, or 1000 msec. Touching the appropriate circle within $2 \mathrm{~s}$ resulted in reinforcer delivery. The time required for the monkey to release the lever (release latency) as well as the time required to touch the circle (response latency) following target onset was recorded in milliseconds. The time required to move from lever to target (movement time) was calculated by subtracting the release latency from the response latency.

Progressive-ratio Schedule of Responding. In the PR task a large colored rectangle was presented in the center of the screen and the animal was required to touch the rectangle for reinforcer delivery. The response requirement started at 1 and incremented by arithmetic progression within blocks of 8 reinforcers and by geometric progression between blocks of 8 . (i.e., the first successive 8 ratios increase by 1 , the second successive 8 increase by 2 , the third successive 8 increase by 4 , etc.) The session was terminated after $10 \mathrm{~min}$, or earlier if 3 min had elapsed since the previous response. In either case, the number of reinforcers acquired in the session was recorded.

Bimanual Motor Skill Task (BMS). A transparent plastic board drilled with 15 holes and filled with raisins was mounted perpendicular to the door of the transport cage. The hole diameter is such that for efficient retrieval of raisins, the animal must push the raisin partially out of the hole with one finger before retrieving it. With training, animals universally adopt a strategy of pushing the raisin with one hand while retrieving it with the other hand, thus entailing bimanual dexterity. The time required to retrieve all 15 raisins was recorded by stopwatch.

\section{Drug Challenge Studies}

Ketanserin tartrate, 1-(3-Chlorophenyl)piperazine dihydrochloride (mCPP) and ( \pm )8-hydroxy-DPAT hydrobromide (8-OH-DPAT) were obtained from RBI (Nat- ick, MA). During the acute drug studies monkeys were tested five days per week with three tasks being completed each day (i.e., SOSS/RT/Bimanual alternated with PR/SOSS/Bimanual). To evaluate the effects of the serotonergic compounds on cognitive/behavioral performance, monkeys were serially administered acute doses of ketanserin $(0.1,0.3,1.0,1.7 \mathrm{mg} / \mathrm{kg})$, $\mathrm{mCPP}(0.03,0.1,0.3,0.5 \mathrm{mg} / \mathrm{kg})$ and 8-OH-DPAT (32, $56,80,100 \mu \mathrm{g} / \mathrm{kg}$,). Doses are expressed as the salt. All compounds were dissolved in physiological saline to a standard volume of $0.1 \mathrm{ml} / \mathrm{kg}$ and were administered intramuscularly, $15 \mathrm{~min}$ prior to the testing session. Drug doses were administered Tuesday and Friday, with the remaining sessions serving as vehicle (Thursday) or baseline (Monday, Wednesday) control sessions. A minimum 2-week washout period was imposed in between the evaluation of each compound to ensure that each animal had returned to stable baseline performance.

\section{Cerebrospinal Fluid and Brain Tissue Evaluation}

CSF (1-2 ml) was collected by percutaneous cisternal puncture using aseptic techniques under ketamine anesthesia $(20 \mathrm{mg} / \mathrm{kg})$. Samples were collected as described previously (Taffe et al. 2001) and, for the present study, at timepoints 9,13 and 16 months after the MDMA regimen. Samples were centrifuged to remove cellular contamination and stored at $-70^{\circ} \mathrm{C}$. CSF concentrations of 5-HIAA were measured by high-performance liquid chromatography with electrochemical detection (HPLC-EC) as previously described (Taffe et al. 2001).

Two MDMA-exposed and two control animals were sacrificed 17 months following the treatment week. The remaining MDMA-exposed subject was sacrificed 20 months following the treatment week. The remaining control animal was treated with the MDMA regimen described for the previously-treated three MDMA animals approximately 20 months following the previous (vehicle) treatment week. This animal was then sacrificed 19 days after the last MDMA injection. The brainstem was separated above the pons and the cerebral hemispheres were divided with a midsaggital cut. Brain sections were then rapidly frozen on powdered dry ice. Regional brain levels of 5-HT and 5-HIAA were determined by HPLC-EC as previously described (Ricaurte et al. 1992).

\section{Data Analysis}

The neuropsychological test battery measures were analyzed using 2-way repeated measures analysis of variance (ANOVA) with a between-subjects factor of treatment group (MDMA-exposed, vehicle control) and a within-subjects factor of drug condition. Separate anal- 
yses were run for each dependent measure and for each drug compound. Dependent measures evaluated for each task were: SOSS, percent correct trials; RT, release latency and movement time; PR, number of reinforcers acquired; BMS, raisin retrieval latency. Preliminary analysis of SOSS and RT data indicated no significant differences based on trial type (SOSS, number of boxes; $\mathrm{RT}$, target duration), and therefore drug effects were analyzed across difficulty condition for these tasks. Initial comparison of the data from vehicle and baseline sessions taken during each of the three studies indicated no differences; thus the baseline and vehicle sessions taken throughout the three studies were averaged (respectively) and employed as comparison conditions for each separate drug challenge analysis. Significant main effects were followed up post hoc with the TukeyKramer procedure including all possible pair-wise comparisons.

The CSF 5HIAA concentrations were analyzed using randomized block ANOVA with a between-subjects factor of treatment group (MDMA-exposed, vehicle control) and a within-subjects factor of time relative to MDMA exposure (pre-treatment, 9, 13 and 16 months post-treatment). The pretreatment values for each animal represent an average of three CSF samples collected no more frequently than monthly (Taffe et al. 2001). Tissue concentrations of 5-HT and 5HIAA in cortical and subcortical regions were each analyzed by analysis of variance with between subjects factors of treatment group (MDMA-exposed, vehicle control) and brain region (cortical analysis: frontal, parietal, temporal and occipital cortex, anterior cingulate, hippocampus; subcortical analysis: caudate (head), putamen, globus pallidus, thalamus, hypothalamus). Post hoc exploration of significant main effects was with the Tukey-Kramer procedure, as above.

All analyses were conducted using GB-STAT v7.0 for Windows (Dynamic Microsystems, Inc., Silver Spring $\mathrm{MD})$. The criterion for significance in all tests was $p<$ .05 and the criterion values were corrected for all possible comparisons in the post hoc tests.

\section{RESULTS}

\section{Self-ordered Spatial Search}

Self-ordered spatial search performance was significantly impaired by mCPP (main effect of drug condition: $\mathrm{F}_{5,20}=3.55, p<.05$ ) and 8-OH-DPAT (main effect of drug condition: $\mathrm{F}_{5,20}=24.08, p<.05$ ) as is illustrated in Figure 1; however, there were no significant effects of ketanserin (main effect of drug condition: $\mathrm{F}_{5,20}=0.40, p=$ .84). There were no significant effects of treatment group. Post-hoc exploration of the effect of $\mathrm{mCPP}$ challenge (across treatment group) confirmed that the 0.5- $\mathrm{mg} / \mathrm{kg}$ dose significantly reduced the percentage of correct trials compared with the $0.03-\mathrm{mg} / \mathrm{kg}$ challenge condition while a similar analysis confirmed that the $100 \mu \mathrm{g} / \mathrm{kg}$ dose of 8-OH-DPAT significantly impaired performance relative to baseline, vehicle and all lower 8-OH-DPAT dose conditions. There were no significant interactions of treatment group with any of the drug challenges; however, post-hoc exploration of all possible pairwise comparisons confirmed that the $100 \mu \mathrm{g} / \mathrm{kg}$ dose of 8-OH-DPAT reduced performance relative to baseline, vehicle and all lower-dose challenges for the control group. The MDMA-exposed animals were similarly affected at the $100 \mu \mathrm{g} / \mathrm{kg}$ dose except that performance at this dose did not differ significantly from the $80 \mu \mathrm{g} / \mathrm{kg}$ dose challenge. Overall, the results suggest that spatial working memory is impaired by stimulation of $5 \mathrm{HT}_{1 \mathrm{~A}}$ and $5 \mathrm{HT}_{2 \mathrm{C}}$ receptors; however, prior exposure to MDMA does not alter monkeys' sensitivity on this measure.

\section{Five-choice Reaction Time}

Release latency in the Reaction Time task was significantly slowed by challenge with $\mathrm{mCPP}$ (main effect of drug condition: $\mathrm{F}_{5,20}=4.25, p<.05$ ) as is illustrated in Figure 2. There were no significant differences attributable to treatment group. Post-hoc exploration of this effect of $\mathrm{mCPP}$ confirmed that the $0.5-\mathrm{mg} / \mathrm{kg}$ dose slowed release latency relative to baseline, vehicle and the 0.03$\mathrm{mg} / \mathrm{kg}$ dose conditions for the MDMA-exposed animals but not for the control group. A trend toward an increase in release latency produced by 8-OH-DPAT failed to reach statistical significance (main effect of drug condition: $\mathrm{F}_{5,20}=2.26, p=.088$ ) and there was no significant effect of ketanserin (main effect of drug condition: $F_{5,20}=$ $1.34, p=.29$ ) on this measure. A trend for the MDMAexposed animals to exhibit slowed release latencies relative to the control animals failed to reach statistical significance in all three drug challenge studies. Movement time was significantly slowed by $\mathrm{mCPP}$ (main effect of drug condition: $\mathrm{F}_{5,20}=3.68, p<.05$ ) but not by ketanserin (main effect of drug condition: $\mathrm{F}_{5,20}=2.21, p=.094$ ) or 8-OH-DPAT (main effect of drug condition: $\mathrm{F}_{5,20}=2.30$, $p=.083$ ), much as was the case for release latency. Similarly, there were no significant differences attributable to treatment group. Post-hoc exploration of the mCPP effect on movement time, however, failed to confirm any significant effects attributable to specific doses either within or across treatment group. Thus, while both release latency and movement time were slowed by $5 \mathrm{HT}_{2 \mathrm{C}}$ stimulation, the MDMA-exposed group exhibited increased sensitivity to $\mathrm{mCPP}$ relative to control animals only in the release latency measure. This result indicates that vigilance may be more easily disrupted in animals previously exposed to MDMA. 


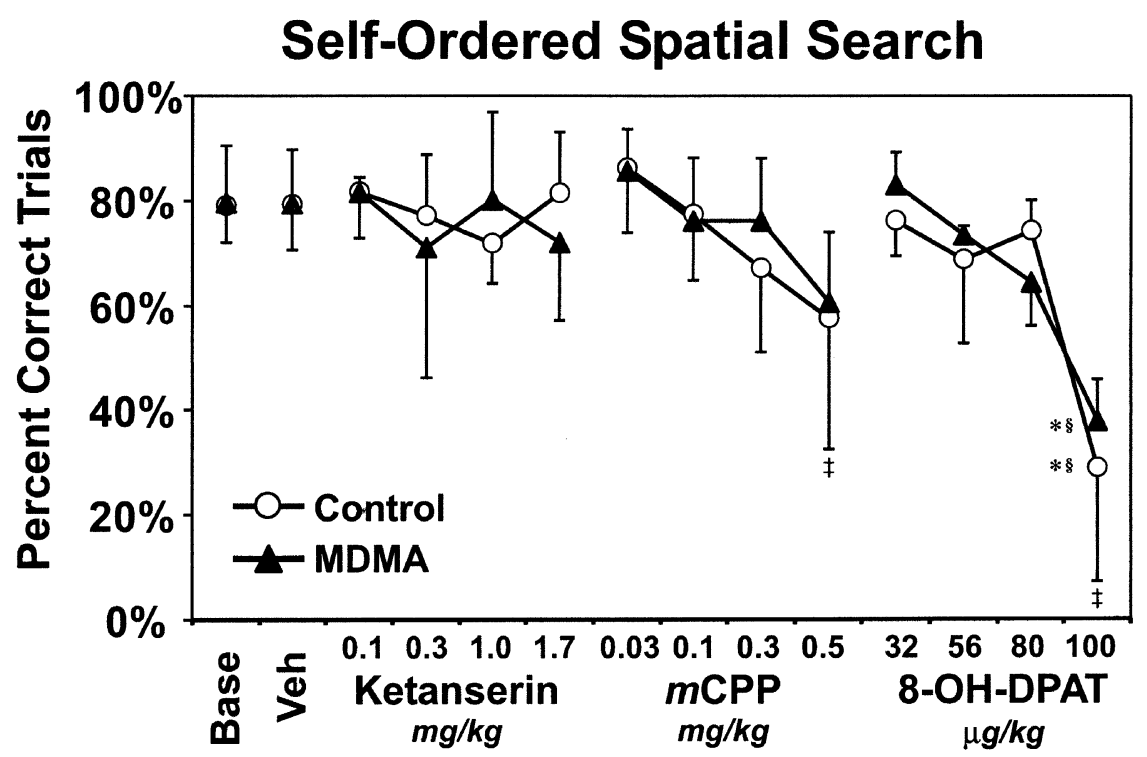

Figure 1. Self-Ordered Spatial Search performance. Mean percentage of correct trials on the SOSS task is presented for MDMA-exposed $(n=3)$ and control $(n=$ 3) monkeys following challenge with three serotonergic agents. Error bars indicate the standard error for each group. Performance on this test of spatial working memory was impaired by challenge with $\mathrm{mCPP}$ and 8-OH-DPAT but not ketanserin for both groups. A significant difference from the lowest dose condition, across treatment group, is indicated by $\ddagger$. For the within-group analysis, * indicates a significant difference compared with the vehicle condition and ${ }^{\S}$ indicates a significant difference compared with the lowest dose administered for a given compound. See text for complete description of the statistical procedures.

\section{Progressive Ratio}

Progressive ratio responding was altered by challenge with 5-HT agents as illustrated in Figure 3, panel A. The number of reinforcers acquired was significantly reduced by $\mathrm{mCPP}$ (main effect of drug condition: $\mathrm{F}_{5,20}=$ $8.29, p<.05$ ) and 8-OH-DPAT (main effect of drug condition: $\left.\mathrm{F}_{5,20}=9.70, p<.05\right)$ but not affected by ketanserin (main effect of drug condition: $\mathrm{F}_{5,20}=1.91, p=$ .14). There were no significant differences attributable to treatment group. Post-hoc analysis of the effects of the drug challenges (collapsed across treatment group) confirmed that the highest dose of mCPP significantly impaired performance relative to baseline, vehicle and the 0.03 and $0.1 \mathrm{mg} / \mathrm{kg}$ doses. Similarly, the highest dose of 8-OH-DPAT impaired performance relative to all other treatment conditions. Although no significant interactions between treatment group and drug condition were observed for any of the drug challenges, further analysis of the main effect of $\mathrm{mCPP}$ and 8-OH-DPAT suggests that the MDMA-exposed animals' performance was more sensitive to challenge with $5 \mathrm{HT}$ agonists. Posthoc analysis of all possible pairwise comparisons confirmed that the $0.5-\mathrm{mg} / \mathrm{kg}$ dose of mCPP significantly reduced PR performance relative to baseline, vehicle and the 0.03 and $0.1-\mathrm{mg} / \mathrm{kg}$ challenge conditions in the MDMA-exposed group only. A similar post-hoc analysis also confirmed that the $100 \mu \mathrm{g} / \mathrm{kg}$ dose of 8-OH-DPAT significantly reduced the number of reinforcers acquired relative to baseline, vehicle and the 32 and $56 \mu \mathrm{g} / \mathrm{kg}$ challenge conditions for the MDMAexposed group, but not the control subjects. Thus, PR performance does not appear to be sensitive to blockade of $5 \mathrm{HT}_{2 \mathrm{~A} / 2 \mathrm{C}}$ receptors but is, however, impaired by both $5 \mathrm{HT}_{1 \mathrm{~A}}$ and $5 \mathrm{HT}_{2 \mathrm{C}}$ stimulation. Prior exposure to MDMA rendered monkeys more sensitive to these drug effects; however, there was only a clear difference in the dose response function of the two groups for the mCPP challenge.

\section{Bimanual Motor Skill}

Bimanual motor skill performance was slowed in a dose-dependent manner by ketanserin (main effect of drug condition: $\mathrm{F}_{5,20}=4.82, p<.05$ ), $\mathrm{mCPP}$ (main effect of drug condition: $\mathrm{F}_{5,20}=8.50, p<.05$ ) and 8-OH-DPAT (main effect of drug condition: $\mathrm{F}_{5,20}=16.14, p<.05$ ), as is illustrated in Figure 3, panel B. There were no significant differences attributable to treatment group. Posthoc analysis of the effect of the drug challenges, across treatment group, confirmed that the highest dose of ketanserin slowed raisin retrieval relative to baseline, vehicle and the $0.1-\mathrm{mg} / \mathrm{kg}$ dose conditions. Similarly, the highest dose of mCPP slowed performance relative to baseline, vehicle and the 0.03 and $0.1-\mathrm{mg} / \mathrm{kg}$ conditions. Finally, the highest dose of 8-OH-DPAT slowed performance relative to all other conditions. Further post-hoc exploration of all possible pairwise comparisons failed to confirm any significant effect of ketanserin within either treatment group; however; the highest dose of 8-OH-DPAT significantly slowed raisin retrieval relative to baseline, vehicle, and the $32 \mu \mathrm{g} / \mathrm{kg}$ dose for both MDMA-exposed and control monkeys. Finally, post-hoc analysis of all pairwise comparisons in the $\mathrm{mCPP}$ study confirmed that the $0.5-\mathrm{mg} / \mathrm{kg}$ dose slowed responding in the MDMA-exposed animals relative to the 0.03 dose. Overall, however, the doseresponse functions for the two groups appear identical for all three serotonergic compounds. Thus, while ketanserin, $\mathrm{mCPP}$, and 8-OH-DPAT all interfered with bimanual motor skills in a dose-dependent manner, the 

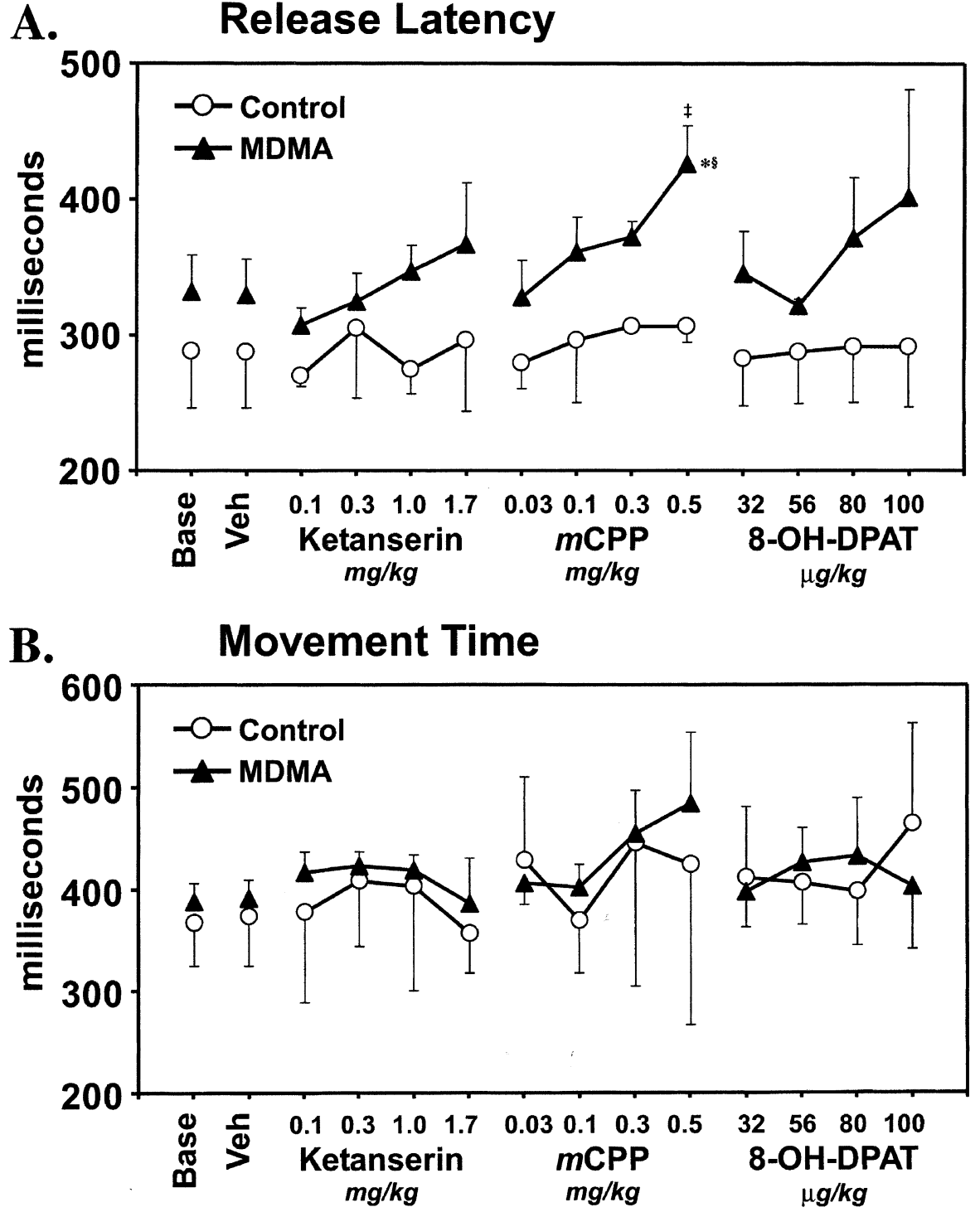

Figure 2. Reaction Time task performance. Mean release latencies (A) and movement times (B) for the RT task are presented for MDMAexposed $(n=3)$ and control $(n=3)$ monkeys following challenge with three serotonergic agents. Error bars indicate the standard error for each group. Challenge with mCPP significantly slowed release latency in the MDMA-exposed monkeys but not the control animals. Neither ketanserin or 8-OH-DPAT had a significant effect on release latency for either group. Movement time was not significantly affected by any of the drug challenges in either group. A significant difference from the lowest dose condition, across treatment group, is indicated by $\ddagger$. For the within-group analysis, * indicates a significant difference compared with the vehicle condition and $\S$ indicates a significant difference compared with the lowest dose administered for a given compound. monkeys previously exposed to MDMA were not more sensitive to the drug effects.

\section{Cerebrospinal Fluid}

CSF samples were obtained at 9, 13, and 16 months following the treatment week. The present acute drug studies were initiated two weeks after the 13-month sample was collected and concluded two weeks prior to the collection of the 16-month sample. As is illustrated in Figure 4, CSF 5-HIAA concentrations for the MDMAexposed animals did not significantly differ from their pre-exposure values nor from the vehicle-treated animals, as demonstrated by a failure to confirm significant main effects of treatment group $\left(\mathrm{F}_{1,4}=0.72, p=\right.$ .45). Similarly, post-treatment CSF 5-HIAA concentrations for the controls did not differ from pre-treatment levels $\left(\mathrm{F}_{3,12}=0.96, p=.44\right)$. These observations are con- sistent with our previous report in which CSF 5-HIAA in this group of MDMA-exposed animals were statistically indistinguishable from pre-treatment values by 21 weeks following treatment (Taffe et al. 2001).

\section{Brain Tissue}

The MDMA treatment regimen produced significant, persistent reductions of 5-HT in cortical regions (Figure 5 ) as confirmed by significant differences between the vehicle and MDMA groups $\left(\mathrm{F}_{1,3}=82.89, p<.05\right)$. The 5 --HT concentration depended on cortical region (main effect of region: $\mathrm{F}_{5,15}=3.90, p<.05$ ) which interacted with treatment group $\left(\mathrm{F}_{5,15}=12.07, p<.05\right)$. Post-hoc exploration of these differences confirmed that there was a persistent depletion of 5-HT in MDMA-exposed animals in frontal, parietal, temporal and occipital cortex, as well as hippocampus, with average regional con- 
A. Progressive Ratio Responding

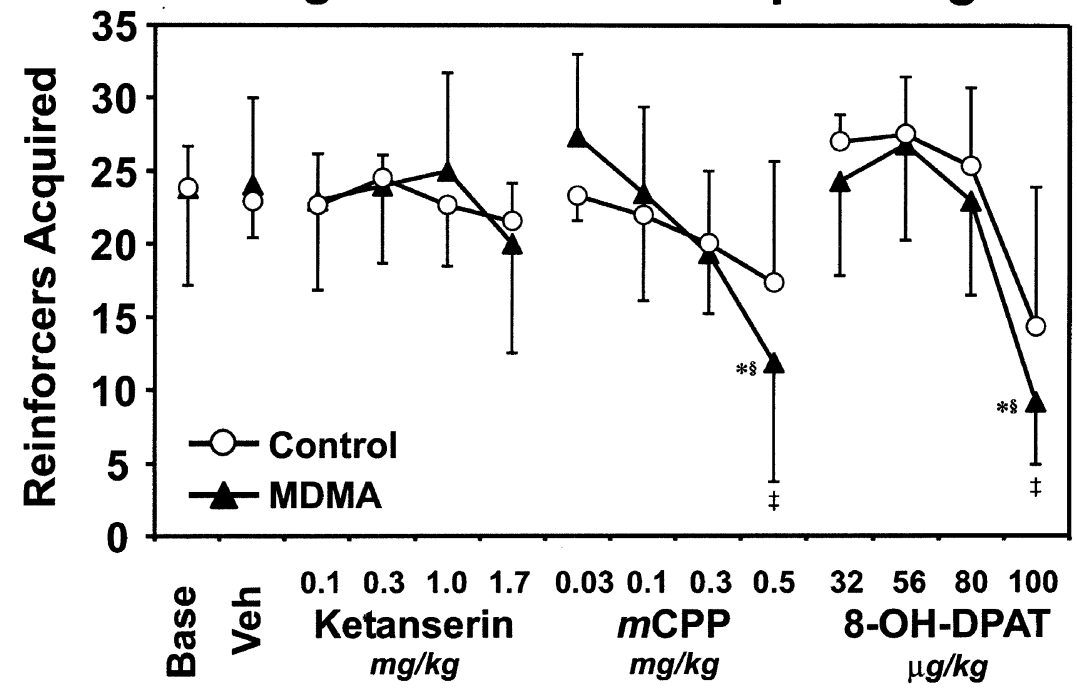

B. Bimanual Motor Skill

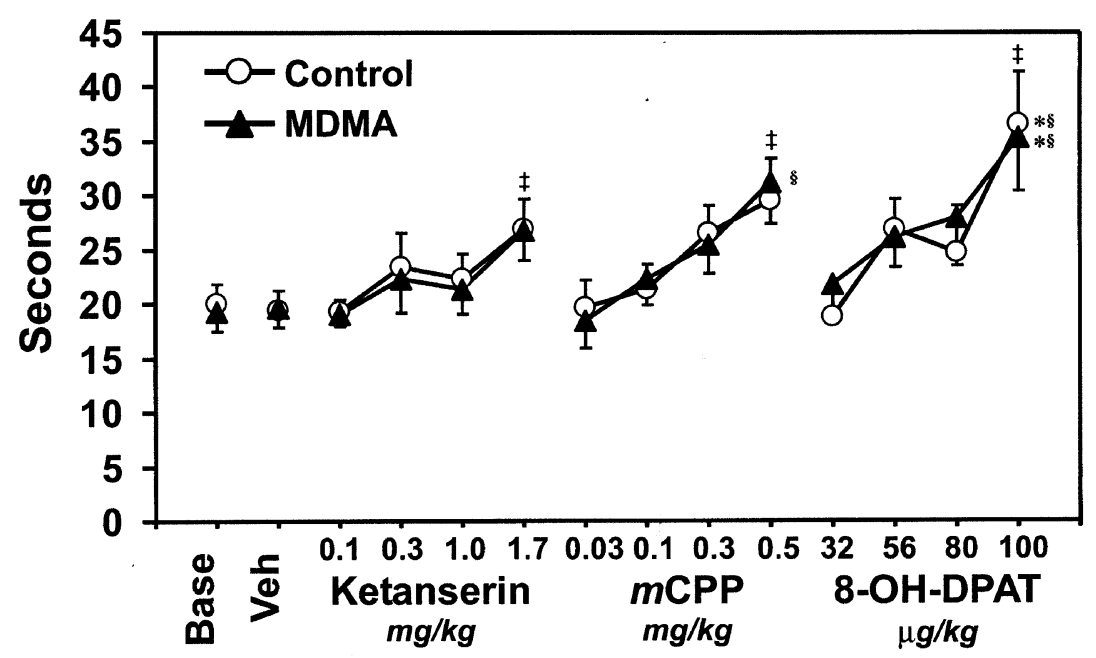

Figure 3. Progressive Ratio responding and Bimanual Motor Skill performance. The mean number of reinforcers acquired on the PR task (A) and the latency to retrieve 15 raisins on the BMS task (B) are presented for MDMAexposed $(n=3)$ and control $(n=3)$ monkeys following challenge with three serotonergic agents. Error bars indicate the standard error for each group. PR performance was significantly decreased by both $\mathrm{mCPP}$ and $8-\mathrm{OH}$-DPAT. The effect of mCPP was more pronounced for the MDMA-exposed monkeys. Raisin retrieval was significantly slowed by all three compounds to an equivalent extent in both groups. A significant difference from the lowest dose condition, across treatment group, is indicated by $\ddagger$. For the within-group analysis, * indicates a significant difference compared with the vehicle condition and $\$$ indicates a significant difference compared with the lowest dose administered for a given compound. centrations reduced by $76 \%$ to $93 \%$ relative to the control animals. There was also a trend for cortical reductions in 5-HIAA with a similar regional pattern (Figure 6) which approached statistical significance $\left(\mathrm{F}_{1,3}=9.47, p=.054\right)$. The MDMA regimen did not result in any statistically significant changes of 5-HT (Figure $5 ; \mathrm{F}_{1,3}=3.45, p=.16$ ) nor 5-HIAA (Figure $6 ; \mathrm{F}_{1,3}=0.96, p=.40$ ) content in the subcortical regions investigated; however, substantial increases in either 5-HT or 5-HIAA content were observed in putamen and globus pallidus.

Thus, these data confirm that the repeated high-dose exposure to MDMA produced depletions of 5HT (and 5 -HIAA) in several regions of neocortex and the hippocampus which persisted for 17-20 months post exposure. These depletions were of a similar magnitude to those observed in a single monkey sacrificed two weeks after exposure to the MDMA regimen. In contrast, there were no lasting depletions observed in subcortical areas examined and possibly even a modest increase observed in putamen and globus pallidus. Since the animal sacrificed only two weeks post-MDMA exhibited substantial reductions in 5HT and 5HIAA in several of the subcortical regions it is likely that the three long-term MDMAexposed animals exhibit a degree of serotonergic recovery, rather than a lack of a primary insult. Overall this pattern of subcortical recovery combined with persistent cortical depletion of 5-HT and 5-HIAA is highly consistent with observations from squirrel monkeys exposed to a similar regimen of MDMA, years prior to sacrifice (Fischer et al. 1995; Hatzidimitriou et al. 1999).

\section{DISCUSSION}

The present investigation was conducted to determine if monkeys exposed to a short-course, high-dose, re- 


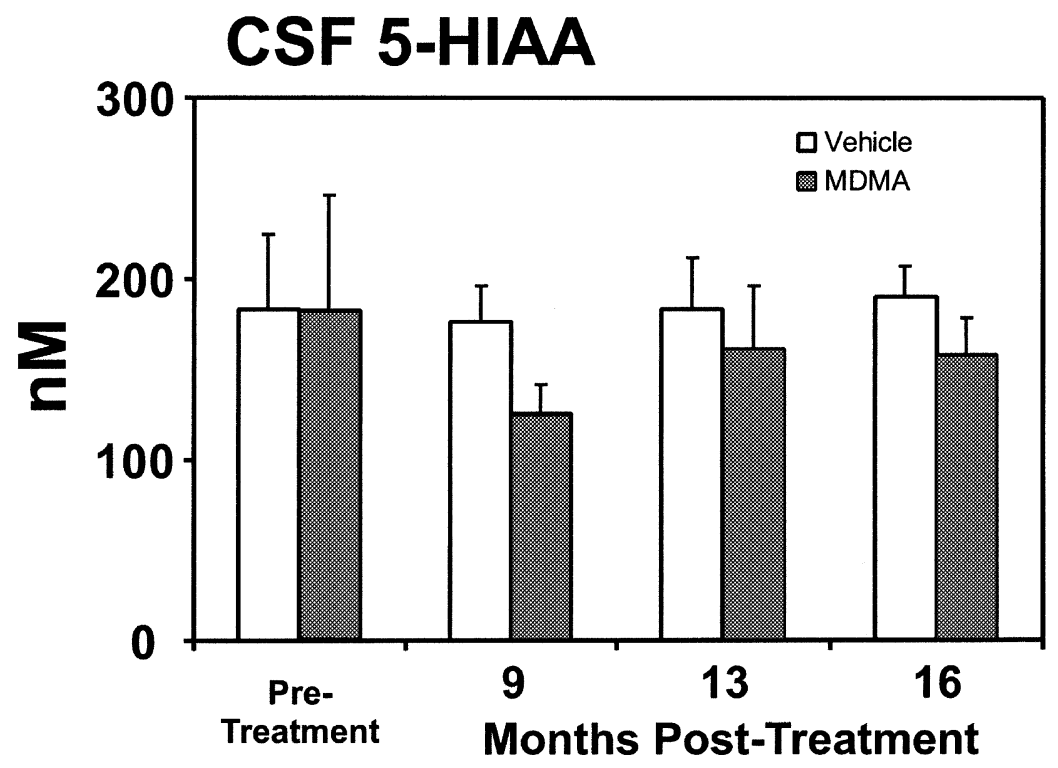

Figure 4. CSF Concentrations of 5-HIAA. Mean concentrations of the 5-HT metabolite 5-HIAA in the CSF of MDMA-exposed ( $\mathrm{n}=$ $3)$ and vehicle-treated control $(n=3)$ monkeys at timepoints 9,13 and 16 months after MDMA exposure, as well as pretreatment values, are presented. Error bars indicate the standard error for each group. The concentrations of 5-HIAA in the CSF of MDMA-exposed monkeys were not significantly different from either control monkeys or their own pre-treatment values at these timepoints. peated regimen of MDMA exhibit lasting behavioral sensitivity to selective pharmacological perturbation of central 5-HT mechanisms. To this end, performance of a number of cognitive/behavioral tasks was challenged serially with doses of the mixed $5 \mathrm{HT}_{2 \mathrm{~A} / 2 \mathrm{C}}$ antagonist ketanserin, the $5 \mathrm{HT}_{2 \mathrm{C}}$ agonist $\mathrm{mCPP}$, and the $5 \mathrm{HT}_{1 \mathrm{~A}}$ agonist 8-OH-DPAT in animals previously exposed to MDMA, as well as a vehicle-treated control group. The

\section{Serotonin}
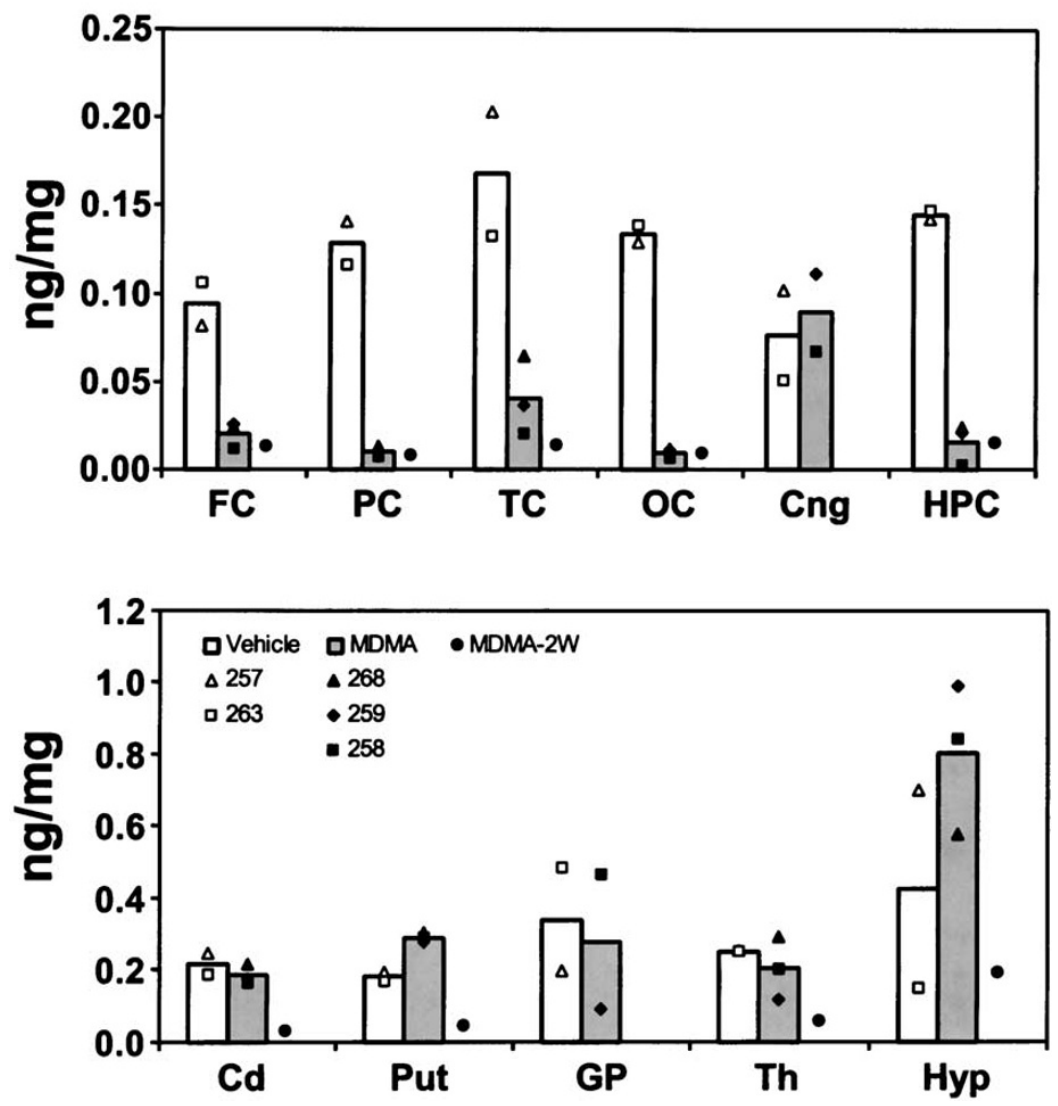

Figure 5. Post-Mortem Tissue Concentrations of 5-HT. Mean and individual concentrations of 5-HT in several brain regions are presented for three animals exposed to the MDMA regimen $17(\mathrm{n}=2)$ or $20(\mathrm{n}=1)$ months prior to sacrifice, and two vehicletreated control animals. Regional concentrations are also presented for one monkey exposed to the repeated MDMA regimen two weeks prior to sacrifice (MDMA-2W; data were not available for the anterior cingulate and globus pallidus of this animal). The MDMA treatment resulted in large, persistent reductions in tissue 5-HT content in all cortical regions except anterior cingulate. The cortical reductions were similar to those observed in the monkey sacrificed two weeks post-MDMA. Tissue concentrations of 5-HT in subcortical regions were not significantly altered 17-20 months following MDMA exposure. FC: Frontal Cortex; PC: Parietal Cortex; TC: Temporal Cortex; OC: Occipital Cortex; Cng: Anterior Cingulate; HPC: Hippocampus; Cd: Caudate; Put: Putamen; GP: globus pallidum; Th: Thalamus; Hyp: Hypothalamus. 
5-HIAA
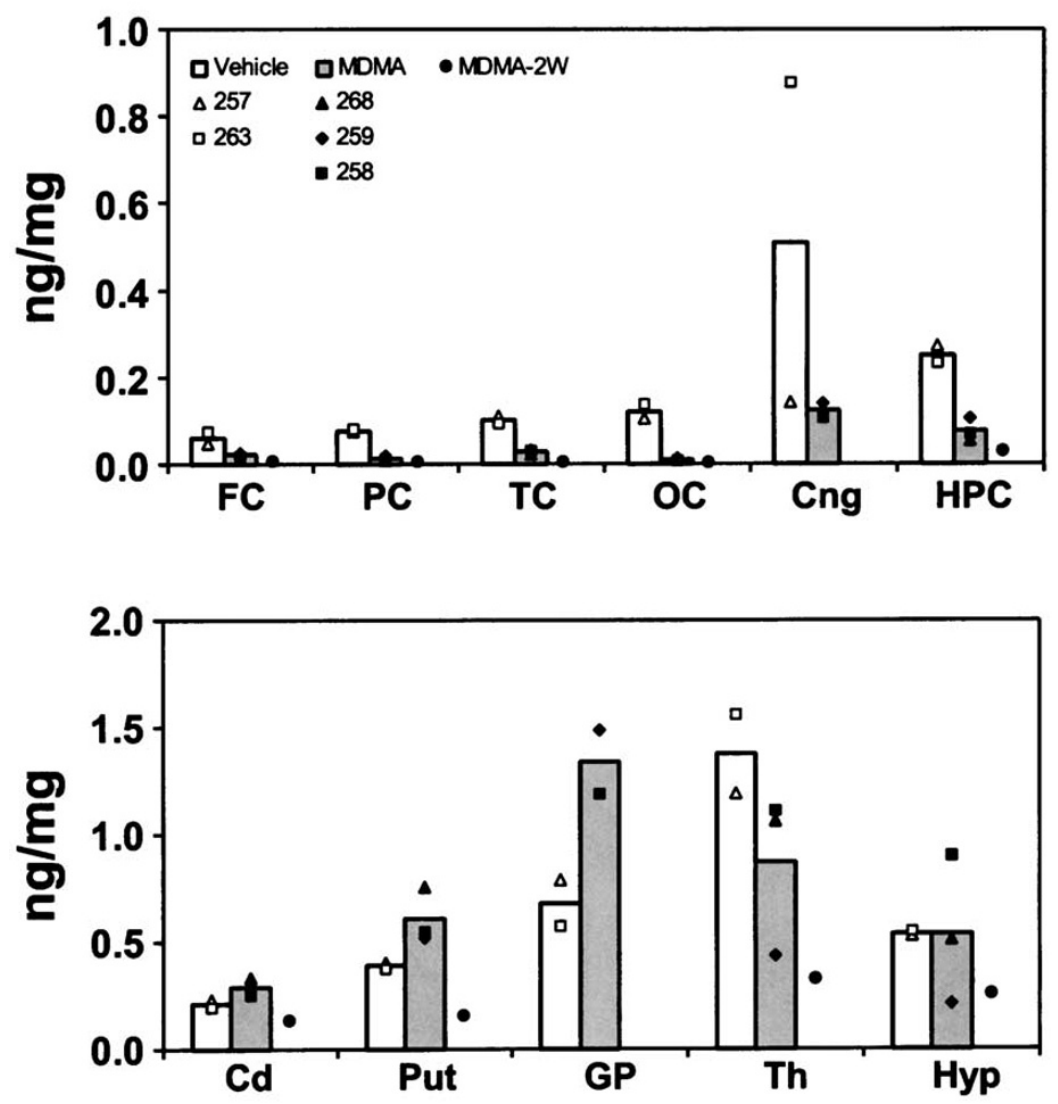

Figure 6. Post-Mortem Tissue Concentrations of 5-HIAA. Mean and individual concentrations of 5-HIAA in several brain regions are presented for three animals exposed to the MDMA regimen 17-20 months prior to sacrifice and two vehicletreated control animals. Regional concentrations are also presented for one monkey exposed to the repeated MDMA regimen two weeks prior to sacrifice (MDMA-2W; data were not available for the cingulate and globus pallidus of this animal). The MDMA treatment resulted in large reductions in tissue 5-HIAA content in all cortical regions except anterior cingulate, consistent with the analysis of 5-HT concentrations shown in Figure 5. Abbreviations of brain regions are as in Figure 5. outcome of this study provides evidence that previous MDMA-exposure may render monkeys more sensitive to serotonergic challenge in some, but not all, cognitive/behavioral domains. Nevertheless, the magnitude of this sensitivity is quite small despite massive $(>75 \%)$ depletions of serotonin content in neocortical regions. Therefore, it is by no means certain that alterations of brain 5-HT systems induced by a single episode of repeated, high-dose exposure to MDMA produce the dramatic alterations of memory and other cognitive functions reported in some human MDMA users.

There are a number of important findings in the present study. First, we have demonstrated that the MDMA-exposed monkeys treated in our previous study, and employed here, exhibit substantial reductions of 5-HT content in several neocortical regions and hippocampus 17-20 months after MDMA treatment. This observation extends the probable duration of persistent 5-HT depletions in rhesus monkeys 3-7-fold over prior studies (Frederick et al. 1998; Insel et al. 1989), and shows similar effects to other reports that 5 -HT depletions in squirrel monkeys persist $1.5-7$ years after MDMA exposure (Fischer et al. 1995; Hatzidimitriou et al. 1999). Furthermore, the pattern of persistent cortical 5-HT depletions combined with recovered (or increased) 5-HT concentrations in subcortical regions was highly consistent with previous observations in squirrel monkeys (Fischer et al. 1995; Hatzidimitriou et al. 1999) in which more exhaustive analyses of brain tissue were conducted, again suggesting that the impact of MDMA on Old World and New World primates is similar, if not identical. Most important for the present study, the post-mortem tissue analyses confirmed that the MDMA-exposed animals were in a state of neocortical 5-HT deficit at the time of the drug challenge studies, despite a failure to observe statistically reliable reductions in CSF 5-HIAA concentrations. Finally, the persistent tissue depletions support our previous conclusions that extensive depletions of 5-HT in the cortex following MDMA-exposure do not result in lasting disruption of cognitive performance, a finding in concordance with two other studies in MDMA-exposed monkeys (Frederick et al. 1998; Winsauer et al. 2002).

Another important finding of the present investigation was that monkeys with $76-93 \%$ reductions of $5-\mathrm{HT}$ in neocortex were more sensitive than control animals to the behaviorally disrupting effects of challenge with serotonergic agents. In particular, there was an increased impact of the highest dose of $\mathrm{mCPP}$ (relative to the lowest dose) on sustained attention and reinforcer efficacy in 
the PR task, and vigilance and response speed in the RT task, for the monkeys previously exposed to MDMA. Similarly, the highest dose of 8-OH-DPAT significantly interfered with PR performance in the MDMA-exposed but not control animals. Finally, although only mCPP caused a statistically significant slowing of release latency in the RT task, there was also an apparent trend for this measure to be more sensitive to both ketanserin and 8-OH-DPAT in the MDMA-exposed monkeys. Overall, however, it must be recognized that the dose-response functions for the two groups overlap substantially for most of the behavioral measures, much as with a recent report on the impact of fenfluramine and $\mathrm{mCPP}$ on repeated acquisition in MDMA-exposed squirrel monkeys (Winsauer et al. 2002).

Taken as a whole, however, the available preclinical data from nonhuman primates tend to suggest that one or two episodes of repeated, high-dose exposure to MDMA are not sufficient to produce obvious disruptions of cognitive or behavioral function, despite producing large and lasting depletions of 5-HT in the neocortex (Frederick et al. 1998; Taffe et al. 2001; Winsauer et al. 2002). Including the present study, these data also show that while MDMA-treated monkeys exhibit increased and decreased sensitivity to challenges of various serotonergic mechanisms, these effects are limited in both magnitude and scope. It should be emphasized, however, that each of the Frederick et al. (1998), Taffe et al. (2001), and Winsauer et al. (2002) studies, as well as the present investigation, employed a treatment group size of $n=3$ which may have resulted in insufficient statistical power to detect real effects of a smaller magnitude. It is possible that larger scale studies might detect additional behavioral or cognitive disturbances. In addition, the Winsauer et al. (2002) study employed only a single behavioral task (with two dependent variables) in contrast to the present investigation and the Frederick et al. (1998) study in which collections of behavioral measures demonstrated persistent alterations in behavioral sensitivity to serotonergic challenges following MDMA exposure. Given that human studies of MDMA users which employ even larger batteries of tests report MDMA-related disturbances of only a few specific measures, it is clear that negative outcomes for particular behavioral measures in the available monkey studies should not be taken as definitive proof of a global lack of cognitive impairment. Nevertheless, interpretations of limited performance disturbance associated with MDMA exposure from the existing data are perhaps not surprising in light of indications that cognitive impairment in human MDMA users is correlated with the duration and severity of MDMA use (Bolla et al. 1998; Gouzoulis-Mayfrank et al. 2000; Morgan 1999; Reneman et al. 2001; Semple et al. 1999) but not with the magnitude of some serotonergic marker abnormalities (McCann et al. 1999; Reneman et al. 2001); but also see
Bolla et al. (1998) and Reneman et al. (2000). Taken as a whole and assuming that the findings from the polydrug-user controlled studies are correct, i.e. that the MDMA use is specifically and causally related to the cognitive impairment, the data suggest that lasting cognitive impairment in MDMA-using populations is likely not a simple function of the magnitude of serotonergic depletions in neocortex. A more plausible alternative is that repeated cycles of serotonergic depletions followed by reinnervation as demonstrated in nonhuman primates (Fischer et al. 1995; Hatzidimitriou et al. 1999), leads to an increasingly abnormal pattern of serotonergic innervation of the CNS. This alternative recommends that future attempts to study the cognitive effects of MDMA exposure in preclinical models employ dosing regimens that more closely match the repeating episodes of human use.

To conclude, the present study demonstrates that monkeys exposed to a single, repeated high-dose MDMA regimen exhibit behavioral sensitivity to selective serotonergic challenge in the absence of any apparent cognitive deficits under normal conditions. Ultimately, such results caution that even recreational MDMA users who appear cognitively/behaviorally intact may exhibit cognitive and behavioral sequelae of MDMA exposure upon perturbation of serotonergic systems.

\section{ACKNOWLEDGMENTS}

We are grateful for the expert technical assistance of Ilham Polis, Tannia Gutierrez, and Bob Lintz. Supported by USPHS grants: DA13390 (MAT), DA11004 (LHP), DA05707 (GAR) and DA09111 (LHG). This is publication \#14556-NP from The Scripps Research Institute.

\section{REFERENCES}

Abraham MD, Cohen PDA, Jan van Til R, Langemeijer MPS (1998): Licit and illicit drug use in Amsterdam III: Developments in Drug Use 1987-1997. Amsterdam, CEDRO

Bhattachary S, Powell JH (2001): Recreational use of 3,4methylenedioxymethamphetamine (MDMA) or 'ecstasy': evidence for cognitive impairment. Psychol Med 31: 647-658

Bolla KI, McCann UD, Ricaurte GA (1998): Memory impairment in abstinent MDMA ("Ecstasy") users [see comments]. Neurology 51:1532-1537

Clark JD, Baldwin RL, Bayne KA, Brown MJ, Gebhart GF, Gonder JC, Gwathmey JK, Keeling ME, Kohn DF, Robb JW, Smith OA, Steggarda J-AD, Vandenbergh JG, White WJ, Williams-Blangero S, VandeBerg JL (1996): Guide for the Care and Use of Laboratory Animals. Washington DC, Institute of Laboratory Animal Resources, National Research Council 
Croft RJ, Mackay AJ, Mills AT, Gruzelier JG (2001): The relative contributions of ecstasy and cannabis to cognitive impairment. Psychopharmacology (Berl) 153:373-379

DeNoble VJ, Schrack LM, Reigel AL, DeNoble KF (1991): Visual recognition memory in squirrel monkeys: effects of serotonin antagonists on baseline and hypoxiainduced performance deficits. Pharmacol Biochem Behav 39:991-996

Fischer C, Hatzidimitriou G, Wlos J, Katz J, Ricaurte G (1995): Reorganization of ascending 5-HT axon projections in animals previously exposed to the recreational drug $(+/-) 3,4-$ methylenedioxymethamphetamine (MDMA, "Ecstasy"). J Neurosci 15:5476-5485

Frederick DL, Ali SF, Gillam MP, Gossett J, Slikker W, Paule MG (1998): Acute effects of dexfenfluramine (d-FEN) and methylenedioxymethamphetamine (MDMA) before and after short-course, high-dose treatment. Ann NY Acad Sci 844:183-190

Gouzoulis-Mayfrank E, Daumann J, Tuchtenhagen F, Pelz S, Becker S, Kunert HJ, Fimm B, Sass H (2000): Impaired cognitive performance in drug free users of recreational ecstasy (MDMA). J Neurol Neurosurg Psychiatry 68:719-725

Hatzidimitriou G, McCann UD, Ricaurte GA (1999): Altered serotonin innervation patterns in the forebrain of monkeys treated with $(+/-) 3,4$-methylenedioxymethamphetamine seven years previously: factors influencing abnormal recovery. J Neurosci 19:5096-5107

Hibell B, Andersson B, Ahlstrom S, Balakireva O, Bjarnason T, Kokkevi A, Morgan M (2000): The 1999 ESPAD Report: Alcohol and other drug use among students in 30 European countries (Summary). The Swedish National Institute of Public Health, Stockholm, Sweden

Insel TR, Battaglia G, Johannessen JN, Marra S, De Souza EB (1989): 3,4-Methylenedioxymethamphetamine ("Ecstasy") selectively destroys brain serotonin terminals in rhesus monkeys. J Pharmacol Exp Ther 249:713-720

Johnston LD, O'Malley PM, Bachman JG (2001a): Monitoring the Future: National Survey Results on Drug Use, 1975-2000. Volume I: Secondary School Students (NIH Publication No. 01-4924). Bethesda, MD, National Institute on Drug Abuse

Johnston LD, O'Malley PM, Bachman JG (2001b): Monitoring the Future: National Survey Results on Drug Use, 1975-2000. Volume II: College Students and Adults Ages 19-40 (NIH Publication No. 01-4925). Bethesda, MD, National Institute on Drug Abuse

McCann UD, Mertl M, Eligulashvili V, Ricaurte GA (1999): Cognitive performance in $(+/-)$ 3,4-methylenedioxymethamphetamine (MDMA, "Ecstasy") users: a controlled study. Psychopharmacology (Berl) 143:417-425

McCann UD, Szabo Z, Scheffel U, Dannals RF, Ricaurte GA (1998): Positron emission tomographic evidence of toxic effect of MDMA ("Ecstasy") on brain serotonin neurons in human beings [see comments]. Lancet 352:1433-1437

McKearney JW (1990): Effects of serotonin agonists on operant behavior in the squirrel monkey: quipazine, MK212, trifluoromethylphenylpiperazine, and chlorophenylpiperazine. Pharmacol Biochem Behav 35:181-185

McKenna DJ, Peroutka SJ (1990): Neurochemistry and neurotoxicity of 3,4-methylenedioxymethamphetamine (MDMA, "Ecstasy"). J Neurochem 54:14-22
Morgan MJ (1999): Memory deficits associated with recreational use of "Ecstasy" (MDMA). Psychopharmacology (Berl) 141:30-36

Morgan MJ (2000): Ecstasy (MDMA): a review of its possible persistent psychological effects. Psychopharmacology (Berl) 152:230-248

Parrott AC (2000): Human research on MDMA (3,4-methylene-dioxymethamphetamine) neurotoxicity: cognitive and behavioural indices of change. Neuropsychobiology 42:17-24

Peroutka SJ (1987): Incidence of recreational use of 3,4-methylenedimethoxymethamphetamine (MDMA, "Ecstasy") on an undergraduate campus [letter]. N Engl J Med 317:1542-1543

Pope HG Jr, Ionescu-Pioggia M, Pope KW (2001): Drug use and life style among college undergraduates: a 30-year longitudinal study. Am J Psychiatry 158:1519-1521

Ramsey M, Spiller J (1997): Drug Misuse Declared: Results of the 1996 British Crime Survey. London, Home Office, RSD

Reneman L, Booij J, Schmand B, van den Brink W, Gunning B (2000): Memory disturbances in "Ecstasy" users are correlated with an altered brain serotonin neurotransmission. Psychopharmacology (Berl) 148:322-324

Reneman L, Lavalaye J, Schmand B, de Wolff FA, van den Brink W, den Heeten GJ, Booij J (2001): Cortical serotonin transporter density and verbal memory in individuals who stopped using 3,4-methylenedioxymethamphetamine (MDMA or "Ecstasy"): preliminary findings. Arch Gen Psychiatry 58:901-906

Ricaurte GA, DeLanney LE, Wiener SG, Irwin I, Langston JW (1988a): 5-Hydroxyindoleacetic acid in cerebrospinal fluid reflects serotonergic damage induced by 3,4methylenedioxymethamphetamine in CNS of nonhuman primates. Brain Res 474:359-363

Ricaurte GA, Forno LS, Wilson MA, DeLanney LE, Irwin I, Molliver ME, Langston JW (1988b): (+/-)3,4-Methylenedioxymethamphetamine selectively damages central serotonergic neurons in nonhuman primates. JAMA 260:51-55

Ricaurte GA, Martello AL, Katz JL, Martello MB (1992): Lasting effects of (+-)-3,4-methylenedioxymethamphetamine (MDMA) on central serotonergic neurons in nonhuman primates: neurochemical observations. J Pharmacol Exp Ther 261:616-622

Ricaurte GA, Yuan J, McCann UD (2000): (+ / -)3,4-Methylenedioxymethamphetamine ("Ecstasy")-induced serotonin neurotoxicity: studies in animals. Neuropsychobiology 42:5-10

Rodgers J (2000): Cognitive performance amongst recreational users of "ecstasy". Psychopharmacology (Berl) 151:19-24

Schuster P, Lieb R, Lamertz C, Wittchen HU (1998): Is the use of ecstasy and hallucinogens increasing? Results from a community study. Eur Addict Res 4:75-82

Semple DM, Ebmeier KP, Glabus MF, O'Carroll RE, Johnstone EC (1999): Reduced in vivo binding to the serotonin transporter in the cerebral cortex of MDMA ("Ecstasy") users. Br J Psychiatry 175:63-69

Steele TD, McCann UD, Ricaurte GA (1994): 3,4-Methylenedioxymethamphetamine (MDMA, "Ecstasy"): pharma- 
cology and toxicology in animals and humans. Addiction 89:539-551

Taffe MA, Weed MR, Davis S, Huitron-Resendiz S, Schroeder R, Parsons LH, Henriksen SJ, Gold LH (2001): Functional consequences of repeated $(+/-) 3,4$-methylenedioxymethamphetamine (MDMA) treatment in rhesus monkeys. Neuropsychopharmacology 24:230-239

Taffe MA, Weed MR, Gold LH (1999): Scopolamine alters rhesus monkey performance on a novel neuropsychological testing battery. Cogn Brain Res 8:203-212

Verkes RJ, Gijsman HJ, Pieters MS, Schoemaker RC, de Visser S, Kuijpers M, Pennings EJ, de Bruin D, Van de Wijngaart G, Van Gerven JM, Cohen AF (2001): Cognitive performance and serotonergic function in users of ecstasy. Psychopharmacology (Berl) 153:196-202

Weed MR, Gold LH (1998): The effects of dopaminergic agents on reaction time in rhesus monkeys. Psychopharmacology (Berl) 137:33-42
Weed MR, Taffe MA, Polis I, Roberts AC, Robbins TW, Koob GF, Bloom FE, Gold LH (1999): Performance norms for a rhesus monkey neuropsychological testing battery: Acquisition and long-term performance. Cogn Brain Res 8:184-201

Winsauer PJ, Bixler MA, Mele PC (1996): Comparison of the effects of typical and atypical anxiolytics on learning in monkeys and rats. J Pharmacol Exp Ther 276: $1111-1127$

Winsauer PJ, McCann UD, Yuan J, Delatte MS, Stevenson MW, Ricaurte GA, Moerschbaecher JM (2002): Effects of fenfluramine, $\mathrm{m}$-CPP and triazolam on repeated-acquisition in squirrel monkeys before and after neurotoxic MDMA administration. Psychopharmacology (Berl) 159: 388-396

Zakzanis KK, Young DA (2001): Memory impairment in abstinent MDMA ("Ecstasy") users: a longitudinal investigation. Neurology 56:966-969 